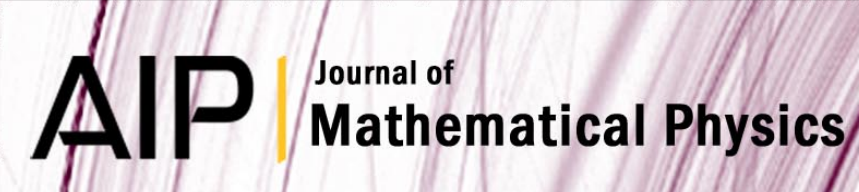 \\ On the representation of twodimensional scalar wave fields in the complex plane
}

M. NietoVesperinas

Citation: J. Math. Phys. 25, 1592 (1984); doi: 10.1063/1.526280

View online: http://dx.doi.org/10.1063/1.526280

View Table of Contents: http://jmp.aip.org/resource/1/JMAPAQ/v25/i5

Published by the American Institute of Physics.

\section{Related Articles}

Using scattering theory to compute invariant manifolds and numerical results for the laser-driven Hénon-Heiles system

Chaos 22, 043138 (2012)

Practical axial optical trapping

Rev. Sci. Instrum. 83, 103106 (2012)

Multiangle static and dynamic light scattering in the intermediate scattering angle range

Rev. Sci. Instrum. 83, 093106 (2012)

Modulated heterodyne light scattering set-up for measuring long relaxation time at small and wide angle Rev. Sci. Instrum. 83, 083102 (2012)

An in-vacuum x-ray diffraction microscope for use in the $0.7-2.9 \mathrm{keV}$ range

Rev. Sci. Instrum. 83, 033703 (2012)

\section{Additional information on J. Math. Phys.}

Journal Homepage: http://jmp.aip.org/

Journal Information: http://jmp.aip.org/about/about_the_journal

Top downloads: http://jmp.aip.org/features/most_downloaded

Information for Authors: http://jmp.aip.org/authors

\section{ADVERTISEMENT}

The most comprehensive support for physics in any mathematical software package World-leading tools for performing calculations in theoretical physics

www.maplesoft.com/physics
Your work in Maple matches how you would write the problems and solutions by hand

- State-of-the-art environment for algebraic computations in physics

The only system with the ability to handle a wide range of physics computations as well as pencil-and-paper style input and textbook-quality display of results

- Access to Maple's full mathematical power, programming language, visualization routines, and document creation tools 


\title{
On the representation of two-dimensional scalar wave fields in the complex plane
}

\author{
M. Nieto-Vesperinas \\ Instituto de Optica, C.S.I.C., Serrano 121, Madrid 6, Spain
}

(Received 16 March 1983; accepted for publication 5 August 1983)

\begin{abstract}
Scalar wave fields satisfying the Helmholtz equation in two dimensions are represented by means of a complex variable associated with the two-dimensional physical plane. This characterizes the wave functions as generalizations of analytic functions, which allows the existence of a generalized Cauchy integral formula constituting the nucleus of well-known theorems of optics such as the theorem of Helmholtz and Kirchhoff and the Ewald-Oseen extinction theorem. It also seems useful in the interpretation of inverse diffraction and scattering problems.
\end{abstract}

PACS numbers: $42.10 . \mathrm{Hc}, 02.30 . \mathrm{Dk}$

\section{INTRODUCTION}

The representation of scalar wave fields by means of functions of a complex variable has an interpretative value in optics. ${ }^{1,2}$ Also, in the interaction of electromagnetic fields with cylindrical scatterers, there is a useful representation of the wave function in the complex plane which permits the location of singularities and analytic continuation of the fields. ${ }^{3,4}$

As in the case of some elliptic equations ${ }^{5,6}$ and, in particular, electrostatic problems, solutions of the two-dimensional Helmholtz equation may be studied in the context of complex variable theory. It will be shown that functions of a complex variable, associated with two-dimensional scalar wave fields, are generalizations of analytic functions. They have a similarity to the generalized analytic functions (in Vekua's terminology $y^{7}$ ), or pseudoanalytic functions (as introduced by Bers $^{8}$ ). In fact, there exists a generalized Cauchy integral formula for those functions, which constitutes the central equation for dealing with boundary-value problems (in the generalized sense of Hilbert). ${ }^{7,9}$ As will be seen, this formula yields a Poisson representation theorem and constitutes the complex variable formulation from which the theorem of Helmholtz and Kirchhoff ${ }^{10,11}$ for scalar wave fields is obtained. It also provides a framework for the interpretation of inverse diffraction problems and other subjects of recent active research such as the extinction theorem of Ewald and Oseen. This theorem, which was initially a result of molecular optics, describing the extinction of an incident wave inside a medium, and its replacement by another wave with different wave number, has recently received a great deal of attention (see, e.g., Refs. 12-14 and references therein). It has been generalized to different media and to quantum potential scattering and has been interpreted by Wolf and Pattanayak as a nonlocal boundary condition for determining fields in the interior of the medium. It will be seen in this paper that the mathematical aspect of the extinction theorem is given by the form adopted by the generalized Cauchy integral formula evaluated at a point exterior to a doubly connected domain.

In the next section we shall establish properties characterizing scalar wave fields in the complex plane associated to the $\mathbb{R}^{2}$ physical plane. This will allow the derivation of the
Cauchy formula (Sec. 3) and its consequences for diffraction and scattering problems (Secs. 4 and 5). The two-dimensional treatment presented here is enough to show the essentials of the ideas that we try to put forward. We believe that a full 3-D treatment, although important from a strict formal point of view when dealing with wave fields in three dimensions, would be much more complicated as it should require the use of quaternionic analysis, ${ }^{15}$ but would not add many more qualitative results, at least up to the scope pursued here.

\section{MATHEMATICAL PRELIMINARIES}

Let us consider a two-dimensional monochromatic scalar field $u(x, y)$ satisfying the Helmholtz equation in a domain $\mathscr{D}$, free of sources, of the $\mathbb{R}^{2}$ plane:

$$
\nabla^{2} u(x, y)+k^{2} u(x, y)=0,
$$

$k$ being the field propagation constant.

Let us associate with the $\mathbb{R}^{2}$ plane the complex $E$ plane by introducing the new variables

$$
z=x+i y, \quad \bar{z}=x-i y
$$

with the operators

$$
\frac{\partial}{\partial \bar{z}}=\frac{1}{2}\left(\frac{\partial}{\partial x}+i \frac{\partial}{\partial y}\right), \quad \frac{\partial}{\partial z}=\frac{1}{2}\left(\frac{\partial}{\partial x}-i \frac{\partial}{\partial y}\right) .
$$

The partial derivatives (3) in a domain $\mathscr{D} \subset E$ must be taken in the generalized sense of Sobolev ${ }^{7}$ or, equivalently, in the areal sense of Pompeiu. ${ }^{9}$ If a continuous function $u(z)$ has these derivatives in $\mathscr{D}$, then that function belongs, respectively, to the manifolds $D_{\bar{z}}(\mathscr{D})$ and $D_{z}(\mathscr{D})$. Also such a function satisfies ${ }^{7}$

$$
\frac{\partial^{p+q} u}{\partial z^{p} \partial \bar{z}^{q}}=\frac{\partial^{p+q} u}{\partial \bar{z}^{q} \partial z^{p}} .
$$

The function $u(x, y)$ satisfying Eq. (1) in $\mathscr{D} \subset \mathbb{R}^{2}$ will satisfy, as a function $u(z)=u_{1}(x, y)+i u_{2}(x, y)$ in $\mathscr{D} \subset E$ the following equation:

$$
\frac{\partial^{2} u(z)}{\partial z \partial \bar{z}}+\frac{k^{2}}{4} u(z)=0 \text {. }
$$

That is, $u(z)$ will belong to the class $D_{\bar{z}}(\mathscr{D})$ and $D_{z}(\mathscr{D})$. Let us consider $u(z)$ as a function of the manifold $D_{\bar{z}}(\mathscr{D})$; then, evi- 
dently, Eq. (5) is equivalent to the pair of equations

$$
\begin{aligned}
& \frac{\partial u(z)}{\partial \bar{z}}= \pm \frac{k}{2} v(z), \\
& \frac{\partial v(z)}{\partial z}=\mp \frac{k}{2} u(z),
\end{aligned}
$$

which are the complex form of the generalized Cauchy-Riemann systems:

$$
\begin{array}{ll}
\frac{\partial u_{1}}{\partial x}-\frac{\partial u_{2}}{\partial y}= \pm k v_{1}, & \frac{\partial u_{2}}{\partial x}+\frac{\partial u_{1}}{\partial y}= \pm k v_{2}, \\
\frac{\partial v_{1}}{\partial x}+\frac{\partial v_{2}}{\partial y}=\mp k u_{1}, & \frac{\partial v_{2}}{\partial x}-\frac{\partial v_{1}}{\partial y}=\mp k u_{2} .
\end{array}
$$

When $k=0$, then $\partial u / \partial \bar{z}=0$, i.e., $u(z)$ becomes analytic in $\mathscr{D}$ and $u(x, y)$ is harmonic.

We shall denote by $\mathscr{U}(\mathscr{D})$ the class of functions $u$ satisfying Eqs. (6) in $\mathscr{D} \subset E$. [We could develop a parallel formalism for the function $v(z)$, but we shall be only interested in the function $u(z)$ as the descriptor of the scalar wave field.] Functions of the class $\mathscr{U}(\mathscr{D})$ have a close relationship with the class of generalized analytic functions in the sense of Vekua ${ }^{7}$ or pseudoanalytic functions as defined by Bers. ${ }^{8}$ In fact, as will be seen, functions of the manifold $\mathscr{U}(\mathscr{D})$ satisfy some theorems that are similar to those established by Vekua and Bers.

Theorem 1: The function $u \in \mathscr{U}(\mathscr{D})$ may be expressed as

$$
\begin{aligned}
& u(z)=\Phi(z) \mp \frac{k}{2 \pi} \iint_{\mathscr{O}} \frac{v(\zeta)}{\zeta-z} d \xi d \eta, \\
& \xi=\xi+i \eta
\end{aligned}
$$

$\Phi$ being an analytic function.

Proof: Since $u(z) \in D_{\bar{z}}(\mathscr{D}), D_{\bar{z}}(\mathscr{D})$ it satisfies the wellknown Green formulas ${ }^{7}$

$$
\begin{aligned}
& \frac{1}{2 i} \int_{\Gamma} u(z) d z=\iint_{\mathscr{D}} \frac{\partial u}{\partial \bar{z}} d x d y, \\
& -\frac{1}{2 i} \int_{\Gamma} u(z) d \bar{z}=\iint_{\mathscr{D}} \frac{\partial u}{\partial z} d x d y,
\end{aligned}
$$

$\Gamma$ being the boundary of $\mathscr{D}$.

Let $z$ be a fixed point of $\mathscr{D}$. Applying Eq. (9a) to the domain $\mathscr{D}_{\epsilon}$, which is the intersection of $\mathscr{D}$ and the domain $|z-\zeta|>\epsilon$, where $\epsilon$ is a sufficiently small positive number, one easily obtains

$$
u(z)=-\frac{1}{2 \pi i} \int_{\Gamma} \frac{u(\zeta) d \zeta}{\zeta-z}-\frac{1}{\pi} \iint_{\mathscr{Q}} \frac{\partial u(\zeta)}{\partial \bar{\zeta}} \frac{d \xi d \eta}{\zeta-z} .
$$

The first term of the right-hand side of Eq. (10) does not depend on $\bar{z}$; hence it satisfies the ordinary Cauchy-Riemann equations, and thus it is an analytic function. Substituting the value of $\partial u / \partial \bar{\zeta}$ given by Eq. (6a) into the second term of Eq. (10), one obtains Eq. (8).

The following theorem is analogous to the reciprocal theorem of Vekua ${ }^{7}$ or the similarity principle of Bers ${ }^{8}$ :

Theorem 2: The function $u(z) \in \mathscr{U}(\mathscr{D})$ may be written as

$$
u(z)=\Phi(z) e^{\omega(z)},
$$

where $\Phi(z)$ is an analytic function of $\omega(z)$ is a continuous function in $\mathscr{D}$ of the class $D_{\bar{z}}(\mathscr{D})$. Moreover, $\omega(z)$ is given by the expression

$$
\omega(z)=\mp \frac{k}{2 \pi} \iint_{\mathscr{D}} \frac{v(\xi) / u(\zeta)}{\zeta-z} d \xi d \eta
$$

Proof: From Eq. (11) one has

$$
\frac{\partial u}{\partial \bar{z}}=u(z) \frac{\partial \omega}{\partial \bar{z}} .
$$

Comparing Eqs. (6a) and (13)

$$
\frac{\partial \omega}{\partial \bar{z}}= \pm \frac{k}{2} \frac{v(z)}{u(z)}
$$

If $z=z_{0}$ is a zero of order $k$ of $u(z)$, then $u(z)$ may be written

$$
\begin{aligned}
& u(z)=A(z)\left(z-z_{0}\right)^{k}, \\
& A\left(z_{0}\right) \neq 0 .
\end{aligned}
$$

But, according to Eq. (6a), from Eq. (15) is obtained

$$
v(z)= \pm \frac{2}{k} \frac{\partial A}{\partial \bar{z}}\left(z-z_{0}\right)^{k}
$$

And from Eq. (16) one has by virtue of Eq. (6a) and (6b)

$$
k \frac{\partial A}{\partial \bar{z}}=-\left[\frac{\partial^{2} A(z)}{\partial z \partial \bar{z}}+\frac{k^{2}}{4} A(z)\right]\left(z-z_{0}\right) .
$$

The right-hand side of Eq. (17) is a function that vanishes at $z=z_{0}$, therefore the quotient

$$
\frac{v(z)}{u(z)}= \pm \frac{2}{k} \frac{1}{A(z)} \frac{\partial A}{\partial \bar{z}}
$$

will vanish at $z=z_{0}\left[A(z) \neq 0\right.$ at $\left.z=z_{0}\right]$.

We conclude, thus, that the right-hand side of Eq. (14) is zero at those points where $u(z)$ is zero. Moreover, we infer from Eq. (14) and Theorem 1 that $\omega(z)$ satisfies Eq. (12).

The representation (11) permits associating to every analytic function $\Phi(z)$ of $\mathscr{D} \subset E$ a function $u(z) \in \mathscr{U}(\mathscr{D})$. Since $e^{\omega(z)}$ is regular and has no zeros, the singularities and zeros of $u(z)$ coincide with the singularities and zeros of $\Phi(z)$. Hence, it follows that if $u(z)$ does not vanish identically, its poles and zeros are isolated and the multiplicity of a zero and the order of a pole are positive integers.

However, unlike analytic and generalized analytic functions, $u(z)$ of the class $\mathscr{U}(\mathscr{D})$ does not satisfy the maximum modulus principle (see also Ref. 16) nor is every uniformly bounded function of the class $u(E)$ a constant, although it satisfies the following theorem:

Theorem 3: Every function $u(z) \in \mathscr{U}(E)$ continuous and uniformly bounded on the whole complex plane $E$ has the form

$$
u(z)=c e^{\omega(z)},
$$

$c$ being a constant.

Proof: By virtue of Liouville's theorem for analytic functions under the hypotheses of this theorem the function $\Phi(z)$ of Theorem 2 will become a constant $c$.

An example of a function $u(z)$ satisfying Theorem 3 is the plane wave given by Eq. (19) with

$$
\begin{aligned}
& \omega(z)=\frac{1}{2} k[(q+i p) z-(q-i p) \bar{z}], \\
& p^{2}+q^{2}=1,
\end{aligned}
$$

$q$ and $p$ being, respectively, the cosine directors of the direc- 
tion of propagation. The function $\omega(z)$ given by $(20)$ satisfies the equations of Theorem 2 and the corresponding $u(z) \in \mathscr{U}(E)$.

Another example of wave functions $u(z)$ satisfying Theorem 3 is given by the class of source-free fields studied by Sherman ${ }^{17}$, which by virtue of Theorem II of Ref. 17 satisfy Eqs. (6) on the whole $E$.

Analogously to Theorem 3, it is straight-forward to prove:

Theorem 4: A function $u(z) \in \mathscr{U}(\mathscr{D})$ which is zero at a set of points of $\mathscr{D}$ with a limit point will vanish identically in $\mathscr{D}$.

As a consequence of Theorem 4 one obtains the following corollary:

Corollary 1: Two functions of the class $\mathscr{U}(\mathscr{D})$ that coincide at a set of points with a limit point in $\mathscr{D}$ are identical in $\mathscr{D}$.

This corollary establishes, in analogy with the theory of analytic functions, that two functions of the class $\mathscr{U}(\mathscr{D})$ that are identical on an arc of $\mathscr{D}$ are identical in the whole $\mathscr{D}$. In fact, as we shall see, there exists a generalized Cauchy integral formula that connects the values of a function $u \in \mathscr{U}(\mathscr{D})$ inside a domain with the values of $u$ on its contour. This leads to the Poisson representation for the Helmholtz equation and constitutes the formulation of the Huygens description of wave field propagation.

\section{THE FUNDAMENTAL KERNELS AND THE GENERALIZED CAUCHY FORMULA}

Let $\Omega_{1}(z, t)$ and $\bar{\Omega}_{2}(z, t)$ be solutions of Eqs. (6). i.e., $\Omega_{1}(z, t)$ and $\Omega_{2}(z, t)$ [complex conjugate of $\bar{\Omega}_{2}(z, t)$ ], satisfy

$$
\begin{aligned}
& \frac{\partial \Omega_{1}}{\partial \bar{z}}= \pm \frac{k}{2} \bar{\Omega}_{2}, \\
& \frac{\partial \Omega_{2}}{\partial \bar{z}}=\mp \frac{k}{2} \bar{\Omega}_{1}
\end{aligned}
$$

and correspond, respectively, to the analytic functions $\Phi_{1}(z)=1 / t-z$ and $\Phi_{2}(z)=0, t$ being a fixed point of $E$. By virtue of Theorem $1, \Omega_{1}$ and $\Omega_{2}$ satisfy the representations

$$
\begin{aligned}
& \Omega_{1}(z, t)=\frac{1}{t-z} \mp \frac{k}{2 \pi} \iint_{\mathscr{D}} \frac{\bar{\Omega}_{2}(\xi, t)}{\zeta-z} d \xi d \eta \\
& \Omega_{2}(z, t)= \pm \frac{k}{2 \pi} \iint_{\mathscr{D}} \frac{\bar{\Omega}_{1}(z, t)}{\zeta-z} d \xi d \eta
\end{aligned}
$$

Evidently, $\Omega_{1}$ and $\Omega_{2}$ hold the following conditions:

$$
\begin{aligned}
& \lim _{z \rightarrow t}(t-z) \Omega_{1}(z, t)=1, \\
& \lim _{z \rightarrow t}(t-z) \Omega_{2}(z, t)=0 .
\end{aligned}
$$

The functions $\Omega_{1}(z, t)$ and $\Omega_{2}(z, t)$ are called the fundamental kernels of the class $\mathscr{U}(\mathscr{D})$.

Now, let us establish the following theorem:

Theorem 5: The function $u(z) \in(\mathscr{D})$ is given at points $z \in E$ from its values at a contour $\Gamma$ enclosing the domain $\mathscr{D}$ by means of the generalized Cauchy integral

$$
\begin{aligned}
& \frac{1}{2 \pi i} \int_{\Gamma}\left[v(\zeta) \bar{\Omega}_{2}(\zeta, z) d \bar{\zeta}-u(\zeta) \Omega_{1}(\zeta, z) d \zeta\right] \\
& \quad=\left\{\begin{array}{lll}
u(z) & \text { when } & z \in \mathscr{D}, \\
\frac{1}{2} u(z) & \text { when } & z \in \Gamma, \\
0 & \text { when } & z \notin \mathscr{D}+\Gamma .
\end{array}\right.
\end{aligned}
$$

Proof: Let $\Gamma$ be a contour of a domain $\mathscr{D}$. It is the union of a finite number of simply smooth closed Jordan curves in which the function $u(z)$ and $\Omega_{1}(z, t), \Omega_{2}(z, t)$ satisfy, respectively, Eqs. (6) and (20) and $\mathscr{D}+\Gamma$ does not contain the point $z=t$; both $u(z) \Omega_{1}(z, t)$ and $v(z) \Omega_{2}(z, t)$ are continuous and belong, respectively, to the manifolds $D_{\bar{z}}(\mathscr{D})$ and $D_{z}(\mathscr{D})$. Hence, the Green formulas, Eqs. (9), may be applied:

$$
\begin{gathered}
\frac{1}{2 i} \int_{\Gamma} u(z) \Omega_{1}(z, t) d z=\iint_{\mathscr{D}} \frac{\partial}{\partial \bar{z}}\left[u(z) \Omega_{1}(z, t)\right] d x d y \\
=\iint_{\mathscr{D}}\left[\Omega_{1}(z, t) \frac{\partial u}{\partial \bar{z}}+u(z) \frac{\partial \Omega_{1}}{\partial \bar{z}}\right] d x d y .
\end{gathered}
$$

And by means of Eqs. (6a) and (20a) one obtains

$$
\begin{aligned}
& \frac{1}{2 i} \int_{\Gamma} u(z) \Omega_{1}(z, t) d z \\
& \quad= \pm \frac{k}{2} \iint_{\mathscr{Z}}\left[u(z) \bar{\Omega}_{2}(z, t)+\Omega_{1}(z, t) v(z)\right] d x d y
\end{aligned}
$$

In a similar fashion, one obtains from Eq. (9b), and by using Eqs. (6b) and (20b)

$$
\begin{aligned}
& -\frac{1}{2 i} \int_{\Gamma} v(z) \bar{\Omega}_{2}(z, t) d \bar{z} \\
& \quad=\mp \frac{k}{2} \iint_{\mathscr{D}}\left[v(z) \Omega_{1}(z, t)+\bar{\Omega}_{2}(z, t) u(z)\right] d x d y .
\end{aligned}
$$

Then, summing (24) and (25) we obtain

$$
\int_{\Gamma}\left[u(z) \Omega_{1}(z, t) d z-v(z) \bar{\Omega}_{2}(z, t) d \bar{z}\right]=0 .
$$

If $t \in \mathscr{D}$, applying Eq. (26) to the domain $\mathscr{D}_{\epsilon}$ bounded by $\Gamma$ and the circle $\Gamma_{\epsilon} \equiv|z-t|=\epsilon$, we get

$$
\begin{aligned}
& \int_{\Gamma}\left[u(z) \Omega_{1}(z, t) d z-v(z) \bar{\Omega}_{2}(z, t) d \bar{z}\right] \\
& \quad-\int_{\Gamma_{\epsilon}}\left[u(z) \Omega_{1}(z, t)-v(z) \bar{\Omega}_{2}(z, t) d \bar{z}\right]=0 .
\end{aligned}
$$

Therefore, when $\epsilon \rightarrow 0$ we obtain by virtue of the conditions (22)

$$
\int_{\Gamma}\left[u(z) \Omega_{1}(z, t) d z-v(z) \bar{\Omega}_{2}(z, t) d \bar{z}\right]=-2 \pi i u(t) .
$$

When $t \in \Gamma$ we obtain an analogous expression in the righthand side of which appear $\pi i u(t)$. In other words, we have derived the generalized Cauchy formula of Eq. (23).

Evidently, when $k=0$ then $\Omega_{1}(\zeta, z)=1 /(z-\zeta)$ and $\Omega_{2}(z, \zeta)=0$, so that Eq. (23) becomes the classical Cauchy formula for analytic functions.

As can be guessed and will be seen later, the fundamental kernels $\Omega_{1}$ and $\Omega_{2}$ are closely related with the Green's function of the Helmholtz equation. As such, we shall look for solutions to Eqs. (20) that satisfy conditions (22), and besides for $z \neq 0, t \rightarrow \infty$,

$$
\Omega_{1}(z, t)=O\left(|t|^{-1 / 2}\right),
$$




$$
\Omega_{2}(z, t)=O\left(|t|^{-1 / 2}\right),
$$

which coincide with the behavior at infinity of cylindrical waves. The solutions to Eqs. (20) subject to the conditions (22) and (28) are

$$
\begin{aligned}
& \Omega_{1}(z, t)= \pm \pi i \frac{k}{2} \frac{|t-z|}{t-z} H_{1}^{(1)}(k|t-z|), \\
& \Omega_{2}(z, t)=\pi i \frac{k}{2} H_{0}^{(2)}(k|t-z|), \\
& |t-z|=[(t-z)(\bar{t}-\bar{z})]^{1 / 2} .
\end{aligned}
$$

$H_{v}^{(1)}$ and $H_{v}^{(2)}$ are respectively, the first and second Hänkel functions of order $v$, whose limiting forms are ${ }^{18}$

$$
\begin{aligned}
& k r \rightarrow 0: \\
& \quad-i H_{0}^{(1)}(k r) \sim i H_{0}^{(2)}(k r) \sim(2 / \pi) \ln (k r), \\
& -i H_{v}^{(1)}(k r) \sim i H_{v}^{(2)}(k r) \sim-(1 / \pi) \Gamma(v)\left(\frac{1}{2} k r\right)^{-v} \\
& \quad(\operatorname{Re} v>0), \\
& k r \rightarrow \infty: \\
& \quad H_{v}^{(1)}(k r) \sim \sqrt{\frac{2}{\pi k r}} e^{ \pm i(k r-v \pi / 2-\pi / 4) .}
\end{aligned}
$$

The two signs in front of the right-hand side of expression (29a) as well as in Eqs. (20) and (6) must be understood in the following way: the upper sign is associated to $H_{1}^{(1)}$ and $H_{0}^{(2)}$ whereas the lower sign is chosen with $H_{1}^{(2)}$ and $H_{0}^{(1)}$. As will be seen, $H_{v}^{(1)}$ and $H_{v}^{(2)}$ are, respectively, associated to outgoing and incoming waves.

It can easily be verified that Eq. (23) can be also applied to an unbounded domain if we impose upon $u(z)$ the additional condition

$$
\frac{|t-z|}{t-z} \frac{\partial u}{\partial \bar{z}} \pm i \frac{k}{2} u=0\left(|z|^{-1 / 2}\right)
$$

for $t$ fixed and $z \rightarrow \infty$. $u(z):$

This condition implies the following asymptotic law for

$$
u(z) \sim \frac{\pi i k}{2} \sqrt{\frac{2}{\pi k|t-z|}} e^{ \pm i|k| t-z \mid-\pi / 4]} .
$$

That is, $u(z)$ vanishes at infinity as fast as a diverging (upper sign) or converging (lower sign) cylindrical wave. Equation (32) is the counterpart in the $E$-complex plane of Sommerfeld radiation condition.

With condition (32), and providing that $u(z)$ satisfies Eqs. (6) in the upper half plane (uhp), $y>0$, Eq. (23) yields a representation for $u(z)$ for $y>0$, in terms of the generalized Cauchy integral on the real axis:

$$
\begin{aligned}
& \frac{1}{2 \pi i} \int_{-\infty}^{\infty}\left[v(\xi) \bar{\Omega}_{2}(\xi, z)-u(\xi) \Omega_{1}(\xi, z)\right] d \xi \\
& \quad= \begin{cases}u(z), & y>0, \\
\frac{1}{2} u(z), & y=0, \\
0, & y<0,\end{cases} \\
& \xi=\xi+i \eta,
\end{aligned}
$$

where the contour $\Gamma$ of Eq. (23) has been chosen to be the real axis and a semicircle in $y>0$ centered at the origin and whose radius $R$ is taken in the limit $R \rightarrow \infty$.

Equation (34) constitutes the Poisson representation for $u(z) \in \mathscr{U}(\mathscr{D}), \mathscr{D}$ being now the upper half of the plane $E$. As such, it can be regarded as an equivalent representation to that of Heins ${ }^{19}$ (see also Ref. 20).

When only $u(\xi)$ is specified on $\eta=0$, subtracting from Eq. (34) the same integral but now evaluated at $\bar{z}$ instead of at $z$ yields

$$
-\frac{1}{\pi i} \int_{-\infty}^{\infty} u(\xi)\left[\Omega_{1}(\xi, z)-\Omega_{1}(\xi, \bar{z})\right] d \xi=u(z), \quad y>0,
$$

which is another Poisson representation equivalent to the Rayleigh-Sommerfeld diffraction integral in the $\mathbf{R}^{2}$-plane since evidently one may obtain from Eq. (29a)

$$
\Omega_{1}(\xi, z)-\Omega_{1}(\xi, \bar{z})=\frac{i}{4} \frac{\partial G}{\partial \mathbf{n}},
$$

$\mathrm{n}$ being the unit normal to $O x$ along the positive $O y$-axis, and $G$ being Sommerfeld's Green function ${ }^{21}$

$G(k|z-\zeta|)= \pm \pi i\left[H_{0}^{(1)}(k|z-\zeta|)-H_{0}^{(2)}(k|\bar{z}-\zeta|)\right]$.

(1)

Let us denote the left-hand side of Eq. (23) by $\Sigma_{\Gamma}^{(2)}(z)$ :

$$
\sum_{\Gamma}^{(2)}(z)=\frac{1}{2 \pi i} \int_{\Gamma}\left[v(\zeta) \bar{\Omega}_{2}(\zeta, z) d \bar{\zeta}-u(\zeta) \Omega_{1}(\zeta, z) d \zeta\right]
$$

The first of Eqs. (23), corresponding to $z \in \mathscr{D}$, is analogous to the theorem of Helmholtz and Kirchhoff in $\mathbb{R}^{2}$ for scalar wave fields. ${ }^{10,11}$ Hence, the generalized Cauchy integral represents for points inside $\mathscr{D}$ the formulation in the $E$-plane of (1) the Huygens-Fresnel description. In fact, $\Sigma_{\Gamma}^{(2)}(z)$ given by Eq. (38) may be written as

$$
\begin{aligned}
\sum_{\Gamma}^{(1)}(z)= & \mp \frac{1}{2} \int_{\Gamma}\left[H_{0}^{(1)}(k|\xi-z|) \frac{\partial u}{\partial \bar{\xi}} d \bar{\xi}\right. \\
& \left.+u(\zeta) \frac{\partial}{\partial \zeta} H_{0}^{(1)}(k|\zeta-z|) d \zeta\right] .
\end{aligned}
$$

By making use of Eqs. (2) and (3), the above takes the form

$$
\begin{aligned}
\sum_{\Gamma}^{(2)}(z)= & \mp \frac{1}{4} \int_{\Gamma}\left[u\left(\nabla H_{0}^{(1)} \cdot d \mathbf{s}+i\left|\nabla H_{0}^{(1)} \times d \mathbf{s}\right|\right)\right. \\
& \left.+H_{0}^{(1)}(\nabla u \cdot d \mathbf{s}-i|\nabla u \times d \mathbf{s}|)\right],
\end{aligned}
$$

$d s$ being the arc element vector of $\Gamma$ taken counterclockwise. Then, $\nabla f \cdot d \mathbf{s}$ and $|\nabla f \times d \mathbf{s}|$ represent, respectively, the components of $\nabla f$ along the tangent $\mathbf{t}$ and the normal $\mathbf{n}$ to $\Gamma^{22}$. Therefore we have

$$
\begin{aligned}
\sum_{\Gamma}^{(1)}(z)= & \mp \frac{1}{4} \int_{\Gamma} \nabla\left(H_{0}^{(2)} u\right) \cdot d \mathrm{~s} \\
& \mp \frac{i}{4} \int_{\Gamma}\left(u \nabla H_{0}^{(1)} \cdot \mathrm{n} d s-H_{0}^{(2)} \nabla u \cdot \mathrm{n} d s\right) .
\end{aligned}
$$

The first integral of Eq. (39) is evidently zero since $\Gamma$ is closed. Thus we are left with 


$$
\sum_{\Gamma}^{(1)}(z)=\frac{1}{4 \pi} \int_{\Gamma}\left(G^{(1)} \frac{(1)}{\partial n}-u \frac{\partial G^{(1)}}{\partial n}\right) d s,
$$

where $G^{(2)}$ denotes the Green's function, respectively, for outgoing and incoming waves,

$$
G^{(1)}(k|\zeta-z|)= \pm \pi i H_{0}^{(1)}(k|\zeta-z|) .
$$

Equation (40) is the Helmholtz-Kirchhoff integral in $\mathbb{R}^{2}$.

\section{BOUNDARY VALUES. THE EXTINCTION THEOREM}

Let us consider a domain $\mathscr{D}^{(-)}$of the $E$-plane containing a wave-field source distribution, and bounded by a contour $\Gamma \cdot \mathscr{D}^{(-)}$is included in a larger domain $\mathscr{D}$ of boundary $\Gamma^{\prime}$. We shall denote by $z_{<}$a point $z \in \mathscr{D}^{(-)}$and by $z_{>}$a point $z \in \mathscr{D}^{(+)}=\mathscr{D}-\mathscr{D}^{(-)}$(Fig. 1). Evidently $u(z)$ satisfies Eqs. (6) outside $\mathscr{D}^{(-)}$, i.e., $u(z) \in \mathscr{U}\left(\mathscr{D}^{(+)}\right)$.

For $z \in \mathscr{D}^{(-)}$, the generalized Cauchy formulas, Eqs. (23) applied to the doubly connected domain $\mathscr{D}^{(+)}$yield

$$
0=\sum_{\Gamma^{\prime}}^{(1)}\left(z_{<}\right)-\sum_{\Gamma}^{(1)}\left(z_{<}\right),
$$

where the notation of Eq. (38) has been used.

Analogously, for $z \in \mathscr{D}^{(+)}$, Eqs. (23) applied to the domain $\mathscr{D}^{(+)}$give us

$$
u\left(z_{>}\right)=\sum_{\Gamma^{\prime}}^{(1)}\left(z_{>}\right)-\sum_{\Gamma}^{(1)}\left(z_{>}\right) .
$$

Note that in (42) and (43) the integrals $\Sigma_{\Gamma^{\prime}}^{(2)}$, (and $\left.\Sigma_{\Gamma}^{(1)}\right)$ involve the values of $u(\xi)$ and $\partial u / \partial \bar{\zeta}$ on $\Gamma^{\prime}$ (and $\Gamma$ ).

In a direct diffraction or scattering problem the values $u\left(z_{>}\right)$are to be determined from $\Sigma_{\Gamma}^{(1)}$ by means of Eq. (43). In fact, by writing the total field as

$$
u(z)=u^{(i)}(z)+u^{(s)}(z),
$$

where $u^{(i)}(z)$ is a field wave function of the class $\mathscr{U}(E)$, i.e., a function that satisfies Eqs. (6) everywhere in $E$. It is usual to identify $\boldsymbol{u}^{(i)}(z)$ either with an incident field or zero according to whether there are in $\mathscr{D}^{(-)}$secondary or primary sources. On the other hand, $u^{(s)}(z)$ represents the field generated by the sources of $\mathscr{Z}^{(-)}$and is usually referred to as the scattered field or the field radiated by the sources at $\mathscr{D}^{(-)} \cdot \mathcal{u}^{(s)}(z)$ satisfies the radiation condition for outgoing waves [Eq. (32) with the upper sign]. Then, it is evident that $\Sigma_{\Gamma^{\prime}}^{(1)}\left(z_{>}\right)$is equal to

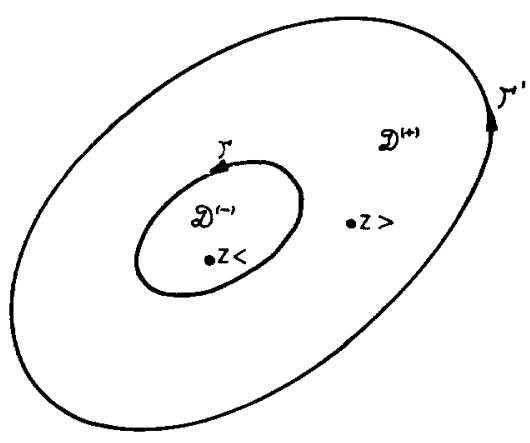

FIG. 1. Contours and domains in the $E$-plane. the value of $\Sigma_{c^{(\infty)}}^{(1)}$, i.e., to the integral (38) with the upper sign over a circle of radius that is taken in the limit tending to infinity. Then we have

$$
\sum_{c^{(\infty)}}^{(1)}=u^{(i)}(z)
$$

since $u^{(i)}$ satisfies the first of Eqs. (23) everywhere in the $E$ plane and the component of $\Sigma_{c^{(\infty)}}^{(1)}$ due to $u^{(s)}(z)$ is zero by virtue of Eq. (32) with the upper sign. Equation (44) is independent of whether $z \in \mathscr{D}^{(-)}$or $z \in \mathscr{D}^{(+)}$.

Thus, Eqs. (42) and (43) yield for the direct problem

$$
\begin{aligned}
& 0=u^{(i)}\left(z_{<}\right)-\sum_{\Gamma}^{(1)}\left(z_{<}\right), \\
& u\left(z_{>}\right)=u^{(i)}\left(z_{>}\right)-\sum_{\Gamma}^{(1)}\left(z_{>}\right) .
\end{aligned}
$$

Eq. (45) may be recognized as the extinction theorem of optics, which constitutes a boundary condition (nonlocal) on $\Gamma$ for $u\left(z_{<}\right) \cdot{ }^{12-14}$ On the other hand, Eq. (46) represents the direct exterior solution. We thus see how both expressions, and in particular the extinction theorem of molecular optics, ultimately are consequences of the generalized Cauchy integral formulas for functions of the class $\mathscr{U}\left(\mathscr{D}^{(+)}\right)$. More specifically, the extinction theorem constitutes the result obtained from the generalized Cauchy formula applied to a point exterior to a doubly connected domain $\mathscr{D}^{(+)}$.

In an inverse diffraction or scattering problem either the values $u(z$, ) or even the values $u(\xi)$ and $\partial u / \partial \bar{\zeta}$ on $\Gamma$ are to be determined from $u(\zeta)$ and $\partial u / \partial \bar{\zeta}$ on $\Gamma^{\prime}$, i.e., from $\Sigma_{\Gamma^{\prime}}^{(2)}$. In this case the choice of the lower superindex of $\Omega_{1}$, and $\Omega_{2}$ in Eqs. (29) indicates that we are considering convergent cylindrical waves and reconstructing the initial values of $u(z)$ from those on a surface $\Gamma^{\prime}$ towards which the wave field $u(z)$ has propagated. In this case Eqs. (42) and (43) yield

$$
\begin{aligned}
& 0=\sum_{\Gamma^{\prime}}^{(2)}\left(z_{<}\right)-\sum_{\Gamma}^{(2)}\left(z_{<}\right), \\
& u\left(z_{>}\right)=\sum_{\Gamma^{\prime}}^{(2)}\left(z_{>}\right)-\sum_{\Gamma}^{(2)}\left(z_{>}\right) .
\end{aligned}
$$

Note that $u(z)$ may be decomposed according to Eq. (44), it satisfies the same radiation condition at infinity and, thus, now $\Sigma_{\Gamma^{\prime}}^{(2)}$, does not become $u^{(i)}$. This is a fundamental difference between direct and inverse problems.

Analogously to the extinction theorem, Eq. (45), the new equation (47) constitutes a nonlocal boundary condition for determining the interior field $u\left(z_{<}\right)$and in particular its value $u(\xi)$ and $\partial u / \partial \bar{\xi}$ on the boundary $\Gamma$ of $\mathscr{D}^{(-)}$. The inverse exterior solution $u\left(z_{<}\right)$is then determined from these values and $\Sigma_{\Gamma}^{(2)}$, by means of Eq. (48).

In the case in which $u\left(z_{>}\right)$represents a source free field ${ }^{17}$ the contribution of $\Sigma_{\Gamma}^{(2)}\left(z_{>}\right)$in Eq. (48) is negligible. This is in agreement, on the other hand, with the fact that if $u\left(z_{>}\right)$is source-free it may be extended into the domain $\mathscr{D}^{(-)}$(Ref. 17) to obtain a bounded solution of Eqs. (6) for all the $E$-plane and, thus, the Cauchy formula for $u(z)$ will apply to any simply connected domain $\mathscr{D}^{(+)} \subset E$, without excluding a domain of sources as in Eq. (48). Hence, the Cauchy integral formula yields for a source-free field the following inverse 
equation instead of Eq. (48):

$$
u\left(z_{>}\right)=\sum_{\Gamma^{\prime}}^{(2)}\left(z_{>}\right) \text {. }
$$

In particular, when $\Gamma^{\prime}$ is chosen as the real axis and a semicircle in $y>0$ centered at the origin and whose radius $R$ is taken in the limit $R \rightarrow \infty$, Eq. (49) becomes the 2-D analogous to the inverse diffraction equation of Shewell and Wolf [Eq. (4.9) of Ref. 23].

In fact the boundary values $\partial u / \partial \zeta$ and $u(\xi)$ may be considered as given on the real axis and any parallel to the real axis in the uhp, $y>0$, by means of a conformal mapping that transforms the $\Gamma$-contour into the line $y=0$. The domain $\mathscr{D}^{(-)}$thus becoming the lhp which contains the sources, and the domain exterior to $\mathscr{D}^{(-)}$being mapped into the uhp free of sources.

\section{THE GENERALIZED CAUCHY FORMULA IN A DOMAIN CONTAINING SOURCES}

We should add to the previous analysis, that it is even possible to obtain a generalized Cauchy formula for a function $u(z)$ in a domain $\mathscr{D}$ containing sources, such as the domains $\mathscr{D}^{(-)}$or $\mathscr{D}$ of Fig. 1.

This is the case for a wave field $u(z)$ satisfying the complex form of the inhomogeneous Helmholtz equation,

$$
\frac{\partial^{2} u(z)}{\partial z \partial \bar{z}}+\frac{k^{2}}{4} u(z)=\frac{1}{4} \rho(z),
$$

$\rho(z)$ denoting the source distribution.

Equation (50) is equivalent to the pair of equations

$$
\begin{aligned}
& \frac{\partial u}{\partial \bar{z}}= \pm \frac{k}{2} v(z), \\
& \frac{\partial v}{\partial z}=\mp \frac{k}{2} u(z) \pm \frac{2}{k} \rho(z) .
\end{aligned}
$$

Of course, the function $u(z)$ satisfying Eqs. $(51)$ is no longer a function of the class $\mathscr{U}(\mathscr{D})$. However, in an analogous way as Theorem 5 was proven, it is easy to derive the following generalized Cauchy formula for $u(z)$ :

$$
\begin{aligned}
\frac{1}{2 \pi i} \int_{\Gamma}\left[v(\zeta) \bar{\Omega}_{2}(\xi, z) d \bar{\zeta}-u(\xi) \Omega_{1}(\zeta, z) d \xi\right] \\
\quad \pm \frac{1}{2 \pi k} \iint_{\mathscr{O}} d x d y \rho(z) \bar{\Omega}_{2}(\xi, z) \\
= \begin{cases}u(z), & z \in \mathscr{D}, \\
\frac{1}{2} u(z), & z \in \Gamma, \\
0, & z \notin \mathscr{D}+\Gamma,\end{cases}
\end{aligned}
$$

$\Gamma$ being the boundary of $\mathscr{D}$.

Equations (52), when used together with Eqs. (42) and (43), may transform contour integrals into domain integrals and, in particular, may be used for solving the inverse interior problem, namely, that of finding the source distributions $\rho(z)$ from the knowledge of $\Sigma_{\Gamma^{\prime}}^{(1)}(z) .^{24,25}$

\section{SUMMARY AND CONCLUSIONS}

We have established a complex variable characterization of scalar wave fields that satisfy the Helmholtz equation in two dimensions. This permits us to obtain fundamental mathematical properties for the wave functions in the com- plex plane associated to the physical $\mathbb{R}^{2}$-plane. Specifically, these functions have been seen to be generalizations of analytic functions with many similar properties; in particular, the possibility of establishing a generalized Cauchy integral formula which is fundamental for solving boundary-value problems. This formula is revealed as the nucleus of classical theorems of optics such as the Helmholtz-Kirchhoff theorem and the Ewald and Oseen extinction theorem, and may also be useful in the formulation of inverse scattering and diffraction problems. The analytic nature of the wave functions, in the general sense used here, seems therefore to be a fundamental characteristic of scattered fields: specifically, it is at the root of the conservation of information in wave propagation (Huygens' principle) as stems from the Cauchy integral.

\section{ACKNOWLEDGMENTS}

The author acknowledges several useful discussions with Dr. A. Méndez.

${ }^{1}$ H. M. Nussenzveig, "Causality and analyticity in optics," in AIP Conference Proceedings 65 (American Institute of Physics, New York, 1981), pp 9-30.

${ }^{2}$ G. Ross, M. A. Fiddy, and M. Nieto-Vesperinas, "The inverse scattering problem in structural determinations," in Inverse Scattering Problems in Optics, edited by H. P. Baltes, Topics in Current Physics 20 (Springer-

Verlag, Berlin, 1980), pp. 15-71; M. A. Fiddy, G. Ross, M. Nieto-Vesperinas, and A. M. J. Huiser, "Encoding of information in inverse optical problems," Optica Acta 29, 23-40 (1982).

${ }^{3}$ R. F. Millar: "Singularities of two-dimensional exterior solutions of the Helmholtz equation,” Proc. Camb. Phil. Soc. 69, 175-188 (1971).

${ }^{4}$ M. Neviere, M. Cadilhac, and R. Petit, "Applications of conformal mappings to the diffraction of E.M. waves by a grating," IEEE Trans. Antennas Propag. AP-21, 37-46 (1973).

${ }^{5}$ I. N. Vekua, New Methods for Solving Elliptic Equations (North-Holland, Amsterdam, 1968)

${ }^{6} \mathrm{C}$. Miranda, Partial Differential Equations of Elliptic Type (Springer-Verlag, Berlin, 1970), $\$ 53$.

${ }^{7}$ I. N. Vekua, Generalized Analytic Functions (Pergamon-Addison Wesley, Reading, MA, 1962)

${ }^{8}$ L. Bers, "Function theoretic aspects of the theory of elliptic partial differential equations," in Methods of Mathematical Physics, by R. Courant and D. Hilbert (Interscience, New York, 1962), Vol. 2, pp. 374 406; L. Bers, "An outline of the theory of pseudoanalytic functions," Bull. Am. Math Soc. 62, 291-331 (1956).

${ }^{9}$ F. D. Gakhov, Boundary Value Problems (Pergamon-Addison Wesley, Reading, MA, 1966), Chap. 5.

${ }^{10} \mathrm{M}$. Born and E. Wolf, Principles of Optics (Pergamon, Oxford, 1975), 5th ed., $\$ 8.3$.

${ }^{11} \mathrm{~J}$. W. Goodman, Introduction to Fourier Optics (McGraw-Hill, San Francisco, 1968), Chap. 3.

${ }^{12}$ D. N. Pattanayak and E. Wolf, "General form and a new interpretation of the Ewald-Oseen extinction theorem," Opt. Commun. 6, 217-220 (1972).

${ }^{13}$ D. N. Pattanayak and E. Wolf, "Scattering states and bound states as solutions of the Schrödinger equation with nonlocal boundary conditions", Phys. Rev. D 13, 913-923 (1976).

${ }^{14} \mathrm{E}$. Wolf, "A generalized extinction theorem and its role in scattering theory," in Coherence and Quantum Optics, edited by L. Mandel and E. Wolf (Plenum, New York, 1973), pp. 339-357.

${ }^{15}$ A. Sudbery, "Quaternionic analysis," Proc. Camb. Phil. Soc. 85, 199-225 (1979).

${ }^{16} \mathrm{C}$. Müller, Foundations of the Mathematical Theory of Electromagnetic Waves (Springer-Verlag, Berlin, 1969).

${ }^{17} \mathrm{G}$. C. Sherman, "Diffracted wavefields expressible by plane-wave expansions containing only homogeneous waves," Phys. Rev. Lett. 21, 761-764 (1968); 21, 1220 (E) (1968); J. Opt. Soc. Am. 59, 697-711 (1969).

${ }^{18} \mathrm{~F}$. W. J. Olver, in Handbook of Mathematical Functions, edited by $\mathbf{M}$. 
Abramowitz and I. Stegun (Dover, New York, 1972), Chap. 9.

${ }^{19}$ A. E. Heins, "On an integral equation in diffraction theory," Compos. Math. 18, 49-54 (1967).

${ }^{20}$ R. F. Millar, "The analytic continuation of solutions to elliptic boundary value problems in two independent variables," J. Math. Anal. Appl. 76, 498-515 (1980).

${ }^{21}$ A. Sommerfeld, Optics (Academic, New York, 1964), \$34.

${ }^{22}$ P. M. Morse and H. Feshbach, Methods of Theoretical Physics (McGraw-
Hill, New York, 1953), Vol. 1, \$4.1.

${ }^{23} \mathrm{~J}$. R. Shewell and E. Wolf, "Inverse diffraction and a new reciprocity theorem," J. Opt. Soc. Am. 58, 1596-1603 (1968).

${ }^{24} \mathrm{~N}$. Bleistein and J. K. Cohen, "Nonuniqueness in the inverse source problem in acoustics and electromagnetics," J. Math. Phys. 18, 194-201 (1977).

${ }^{25}$ N. N. Bojarski, "Inverse scattering inverse source theory," J. Math. Phys. 22, $1647-1650$ (1981). 